\title{
Carbon-lon Beam Irradiation and the miR-200c Mimic Effectively Eradicate Pancreatic Cancer Stem Cells Under in vitro and in vivo Conditions
}

\author{
Sei Sai $\mathbb{D}^{\prime}$ \\ Eun Ho Kim² \\ Woong Sub Koom ${ }^{3}$ \\ Guillaume Vares ${ }^{4}$ \\ Masao Suzuki' \\ Shigeru Yamada ${ }^{5}$ \\ Mitsuhiro Hayashi $\mathbb{1 D}^{6}$ \\ 'Department of Charged Particle \\ Therapy Research, Institute for Quantum \\ Medical Science, National Institutes for \\ Quantum and Radiological Science and \\ Technology (QST), Chiba, Japan; \\ ${ }^{2}$ Department of Biochemistry, School of \\ Medicine, Daegu Catholic University, \\ Nam-gu, Daegu, 42472, South Korea; \\ ${ }^{3}$ Department of Radiation Oncology, \\ Yonsei Cancer Center, Yonsei University \\ College of Medicine, Seoul, South Korea; \\ ${ }^{4}$ Institute of Radioprotection and Nuclear \\ Safety (IRSN), Fontenay-aux-Roses \\ Cedex, France; ${ }^{5} \mathrm{QST}$ Hospital, National \\ Institutes for Quantum and Radiological \\ Science and Technology (QST), Chiba, \\ Japan; ${ }^{6}$ Breast Center, Dokkyo Medical \\ University Hospital, Tochigi, 321-0293, \\ Japan
}

Correspondence: Sei Sai

Department of Charged Particle Therapy

Research, Institute for Quantum Medical

Science, National Institutes for Quantum

and Radiological Science and Technology

(QST), 4-9-I Anagawa Inage-ku, Chiba,

Chiba, 263-8555, Japan

Tel +8I-43-206-323 I

Fax +8I 43-206-4I 49

Email sai.sei@qst.go.jp

Eun Ho Kim

Department of Biochemistry, School of

Medicine, Daegu Catholic University, 33

I7-gil, Duryugongwon-ro, Nam-gu,

Daegu, South Korea

Tel + $8253-650-4480$

Email ehI40149@cu.ac.kr
Purpose: The study investigated the molecular mechanisms that killed pancreatic cancer cells, including cancer stem cells (CSCs), by carbon ion beam irradiation alone or in combination with miRNA-200c under in vitro and in vivo conditions.

Methods: Human pancreatic cancer (PC) cells, PANC1 and PK45, were treated with carbon-ion beam irradiation alone or in combination with microRNA-200c (miR-200c) mimic. Cell viability assay, colony and spheroid formation assay, quantitative real-time PCR analysis of apoptosis-, autophagy-, and angiogenesisrelated gene expression, xenograft tumor control and histopathological analyses were performed.

Results: The cell viability assay showed that transfection of the miRNA-200c (10 nM) mimic into pancreatic CSC (CD44+/ESA+) and non-CSC (CD44-/ESA-) significantly suppressed proliferation of both types of cell populations described above. Combining carbon-ion beam irradiation with the miRNA-200c mimic significantly reduced the colony as well as spheroid formation abilities compared to that observed with the treatment of carbon-ion beam alone or X-ray irradiation combined with the miRNA$200 \mathrm{c}$ mimic. Moreover, the combination of carbon ion beam irradiation and miRNA-200c mimic increased the expression of apoptosis-related gene BAX, autophagy-related genes Beclin-1 and p62, addition of gemcitabine (GEM) further enhanced the expression of these genes. In vivo data showed that carbon-ion beam irradiation in combination with the miRNA-200c mimic effectively suppressed xenograft tumor growth and significantly induced tumor necrosis and cavitation.

Conclusion: The combination of miRNA-200c mimic and carbon ion beam irradiation may be powerful radiotherapy that significantly kills pancreatic cancer cells containing CSCs and enhances the effect of carbon-ion beam irradiation compared to carbon-ion beam irradiation alone.

Keywords: pancreatic cancer stem cell, miR-200c, carbon-ion beam

\section{Introduction}

Pancreatic cancer (PC) is one of the most aggressive cancers. It is difficult to improve its prognosis, especially for locally advanced pancreatic cancer (LAPC) despite remarkable medical improvement. ${ }^{1-3}$ Surgical resection is one of the major treatments for LAPC, but most patients experience recurrence and metastasis, resulting in a 5 -year survival rate of less than $20 \% .{ }^{4,5}$ Recent reports indicate that stereotactic body radiotherapy (SBRT), intensity modulated radiation therapy (IMRT), or proton beam radiation therapy alone or in combination with 
chemotherapy resulted in relatively good outcome. However, for the nonresectable cases, the 2-year survival rate is around $20-30 \% .^{6-14}$

Over the past 25 years, more than 600 patients with LAPC have been treated with carbon-ion radiotherapy (CIRT) at the National Institute of Radiological Sciences (NIRS) and patients treated with CIRT along with gemcitabine (GEM) achieved promising outcomes. However, the 2-year overall survival rate was only around $50 \%{ }^{15-18} \mathrm{We}$ have previously reported that carbon-ion beams combined with GEM predominantly destroyed pancreatic cancer stem cells (CSCs). ${ }^{19}$ On the other hand, accumulating evidence indicates that microRNAs (miRs) play a pivotal role in cancer and function as tumor suppressors and oncogenes. ${ }^{20-24}$ In another recent report, we demonstrated that carbon-ion beam irradiation combined with miR-34 mimic and/or rapamycin or miR-29b effectively eradicated high-grade chondrosarcoma CSCs and osteosarcoma cells via the mTOR-FOXO3 axis and the PTEN/AKT-SP1 pathway. ${ }^{25,26}$ miR-200c, known as a tumor suppressor, is involved in inhibiting tumor growth and metastasis by suppressing the CSCs, this process is thought to be closely related to drug resistance and recurrence. ${ }^{27-35}$ Thus, it is very important to develop a strategy to eliminate CSCs efficiently. ${ }^{36-39}$ Recent reports indicate that miR-200c enhances radiosensitivity in several cancer types such as breast, lung, head and neck cancers. ${ }^{40-52}$ Our recent findings demonstrated that high linear energy transfer (LET) carbonion beam irradiation alone or in combination with chemotherapy or molecular targeted agents effectively enhanced the CSC-killing effects in several cancer cell types. ${ }^{52-57}$ Therefore, we hypothesized that the addition of the miRNA-200c mimic may enhance the carbon-ion beam radiosensitivity.

Thus, we explored the molecular mechanism of killing the pancreatic cancer cells, including CSC, by carbon-ion beam irradiation alone or in combination with miRNA-200c under in vitro and in vivo conditions.

\section{Materials and Methods Cell Culture}

PANC1 and PK45 cells were obtained from the American Type Culture Collection. The unsorted cells were cultured in Dulbecco's Modified Eagle medium (DMEM) with $10 \%$ heat-inactivated fetal bovine serum (Biological Industries, Beit-Haemek, Israel), and 100 units $/ \mathrm{mL}$ penicillin, $100 \mu \mathrm{g} / \mathrm{mL} \quad$ streptomycin (Invitrogen) supplemented with $5 \% \mathrm{CO} 2$ at $37^{\circ} \mathrm{C}$. The medium was changed every 3 days. CSCs and nonCSCs isolated from PANC1 and PK45 cells were cultured in the PromoCell Cancer Stem Cell medium (PromoCell, Heidelberg. Germany).

\section{Reagents}

The antibodies used in the present study were as follows: mouse anti-human CD44-PE (BD, Biosciences, USA), mouse anti-human CD326-FITC (EpCAM/ESA, Miltenyi Biotec, Germany), and gemcitabine (GEM). Gemcitabine (Gemzar) was purchased from Eli Lilly Japan K.K., Kobe, Japan. A stock solution of gemcitabine (1 $\mathrm{mM}$ in PBS) was diluted to appropriate concentrations just before use.

\section{Irradiation}

Cells were treated with a $290 \mathrm{MeV} /$ neutron carbon-ion beam with a 6-cm spread-out Bragg peak (SOBP), which was produced by the heavy ion medical accelerator in Chiba (HIMAC) at the National Institutes for Quantum and Radiological Science and Technology (QST) in Japan. ${ }^{30}$ As a reference, cells were exposed to conventional $200 \mathrm{kVp} \mathrm{X}$-ray irradiation (TITAN-320, GE Co., USA). Cells were irradiated with 2, 4, or 6 Gy of X-rays or carbon-ion beams at 1,2 , or 3 Gy.

\section{Transient Transfection of the miR-200c Mimic}

$m i R-200 c$ mimic and the control were purchased from Bioneer Corporation (Daejeon, South Korea). Cells were transfected with $10 \mathrm{nM}$ control or the miR-200c mimic using the miRNA Transfection Reagent Lipofectamine 2000 (Invitrogen, Carlsbad, CA) according to the manufacturer's instructions.

\section{Colony Formation and Spheroid Formation Assays}

Cells were treated with the $m i R-200 c$ mimic for $24 \mathrm{~h}$ and then incubated for 7-9 days. The colonies were then fixed with methanol, stained with $0.2 \%$ methylene blue (SigmaAldrich, St. Louis, MO), and counted. A spheroid formation assay of the CD44+/ESA+ and CD44-/ESA- ell populations sorted from the PANC1 and PK45 cells was performed as described previously. ${ }^{20}$ Briefly, 3000 cells per well were plated in low cell adhesion 96-well plates 
(SUMILON, Sumitomo Bakelite, Tokyo, Japan) for 1 week and the sphere area size was estimated. Data are displayed as average size after 1 week of incubation using the WinROOF 5.6 software (Mitani Corporation, Tokyo, Japan).

\section{Cell Viability Assay}

The CellTiter-Glo Luminescent Cell Viability Assay (Promega), a method that determines the number of viable cells in the culture based on the quantification of ATP present, was used to estimate the cell viability. In the homogeneous assay, a single reagent (CellTiterGlo ${ }^{\circledR}$ Reagent) was added directly to the cells grown in the serum-supplemented medium. Cell viability was also tested by the Trypan Blue exclusion test, in which dead or dying cells were stained, based on the principle that living cells exclude trypan blue dye and do not stain.

\section{Real-Time RT PCR Analysis for the Expression of Various Genes Related to Apoptosis and Autophagy}

RNA was extracted using the Qiagen RNeasy kit, and cDNA was prepared using the $\mathrm{RT}^{2}$ First-Strand Kit (SABiosciences, Frederick, Maryland, USA). The expression of apoptosis, autophagy-related genes was analyzed using the LightCycler ${ }^{\circledR} 96$ real-time PCR system (Roche, Basel, Switzerland). For data analysis, the $\Delta \Delta \mathrm{Ct}$ method was applied using the RT PCR software package and statistical analysis was performed as described previously. ${ }^{26,27}$ The primer sequences used in this study are shown in Table 1. GAPDH was used as a housekeeping gene.

\section{In vivo Xenograft Tumor Control Assay After Carbon-Ion Beam Irradiation Alone} or in Combination with miR-200c Mimic

PK45 cells were injected into the right leg of NOD-SCID mice.

A total 28 NOD-SCID male mice (6-8 weeks) were used in this study. When the xenograft tumor size reached an approximate size of $10 \mathrm{~mm}$, the mice were treated with a carbon-ion beam alone or in combination with miR-200c mimic. The miR-200c mimic was delivered directly to the tumor twice a week for 2 weeks with an in vivo-jet PEI
Table I The Primer Sequences for Real-Time PCR

\begin{tabular}{|c|c|}
\hline Gene & Primer Sequence \\
\hline \multicolumn{2}{|l|}{ GAPDH } \\
\hline Forward & 5'-TGAACGGGAAGCTCACTGG-3' \\
\hline Reverse & 5'-TCCACCACCCTGTTGCTGTA-3' \\
\hline \multicolumn{2}{|l|}{ Bax } \\
\hline Forward & 5'-CAAACTGGTGCTCAAGGCC-3' \\
\hline Reverse & 5'-GCACTCCCGCCACAAAGAT-3' \\
\hline \multicolumn{2}{|l|}{$\mathrm{BCl}-2$} \\
\hline Forward & 5'-ATGTGTGTGGAGAGCGTCAACC-3' \\
\hline Reverse & 5'-TGAGCAGAGTCTTCAGAGACAGCC-3' \\
\hline \multicolumn{2}{|l|}{ Beclin- $I$} \\
\hline Forward & 5'-AGCTGCCGTTATACTGTTCTG-3' \\
\hline Reverse & 5'-ACTGCCTCCTGTGTCTTCAATCTT-3' \\
\hline \multicolumn{2}{|l|}{ p62 } \\
\hline Forward & 5'- GTGAATTCGCTCGCCGCTCGCTAT-3' \\
\hline Reverse & 5'- CGTCTCGAGTGCCTGCTGACAACACCTA -3' \\
\hline \multicolumn{2}{|l|}{ HIFIa } \\
\hline Forward & 5'-CTATGGAGGCCAGAAGAGGGTAT -3' \\
\hline Reverse & 5'-CCCACATCAGGTGGCTCATAA -3' \\
\hline \multicolumn{2}{|l|}{ VEGF } \\
\hline Forward & 5'-CTTGTTCAGAGCGGAGAAAGC T-3' \\
\hline Reverse & 5'-ACATCTGCAAGTACGTTCGTT -3' \\
\hline
\end{tabular}

reagent after carbon ion beam irradiation according to the protocol (Polyplus-Transfection, Strasbourg, France). Briefly, a total $10 \mu \mathrm{g}$ of miR-200c mimic was diluted in $10 \%$ glucose in $\mathrm{ddH} 2 \mathrm{O}$ in one microtube, and in the other microtube, $1.2 \mu \mathrm{L}$ in vivo-jetPEI-Gal $(\mathrm{N} / \mathrm{P}=6)$ was diluted in $10 \%$ glucose in ddH2O. Then, the tubes were mixed and incubated for $15 \mathrm{~min}$ at room temperature (RT). Finally, the solutions containing miR-200c mimic were injected intratumorally. For histopathological analysis, hematoxylin and eosin (HE) staining were performed using formalin-fixed tumor tissue. All animal experiments were conducted in accordance with QST-NIRS institutional animal welfare guidelines and approved by Animal Experiment Ethics Committee (approval number:17-20082, QST).

\section{Statistical Analysis}

One-way analysis of variance (one-way ANOVA) and Bonferroni multiple comparison tests were used to the mean differences between the groups using the 
A
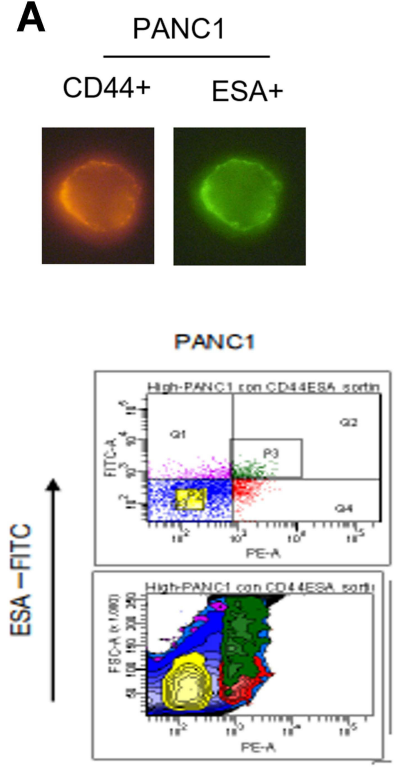

CD44-PE

C

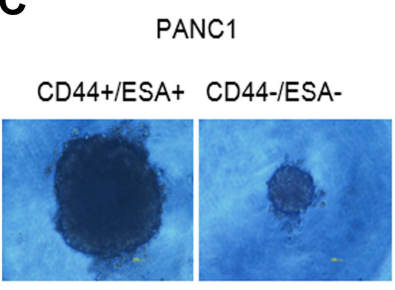

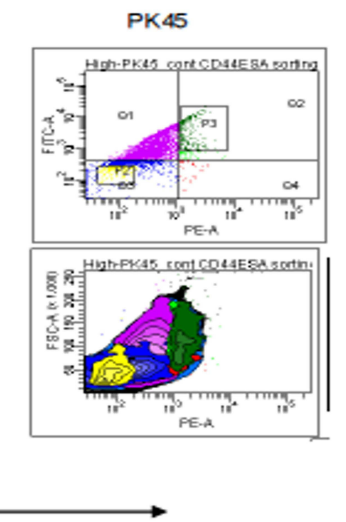
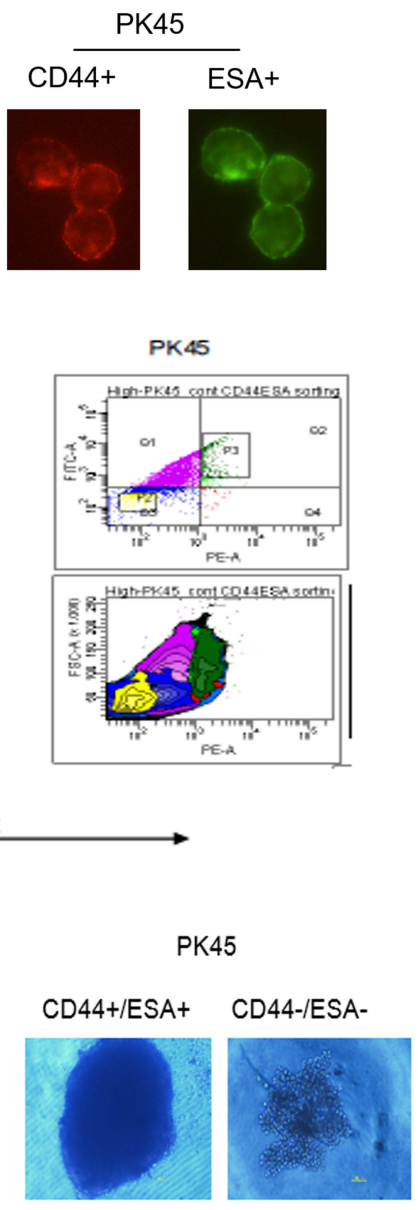

B

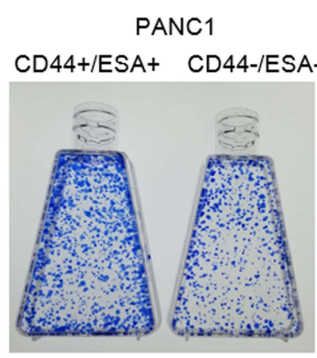

PK45
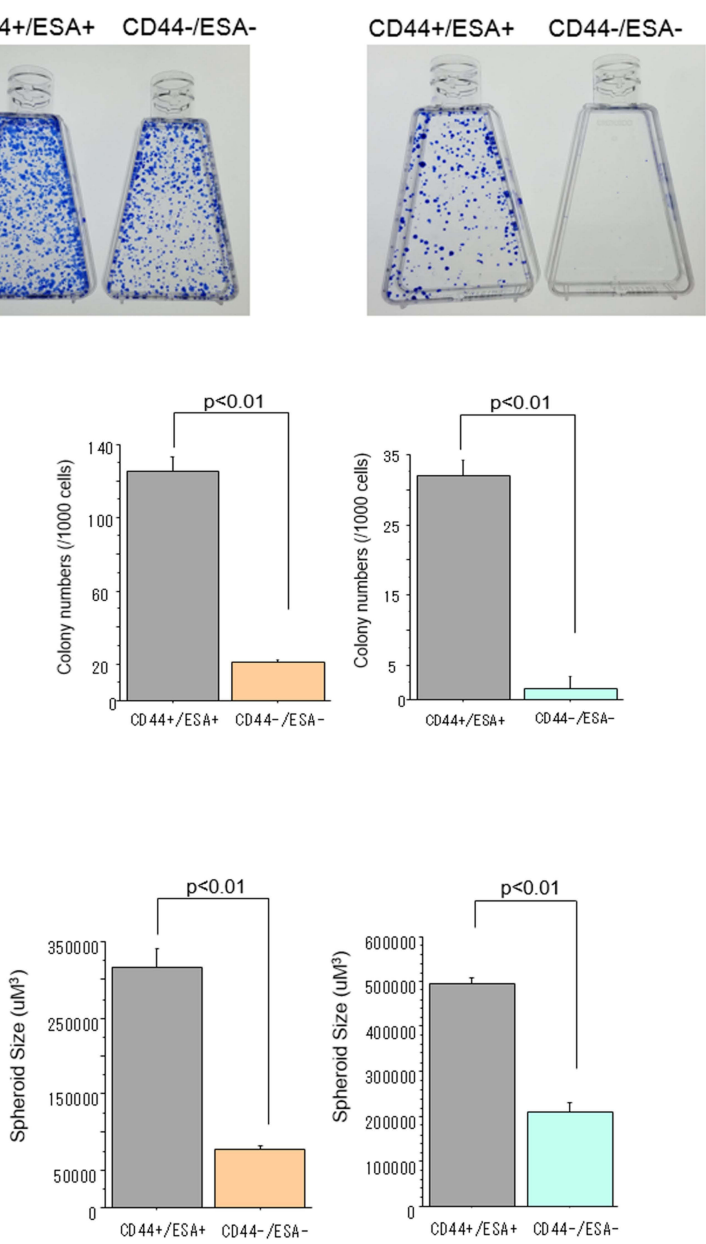

Figure I (A) Representative photos of CD44+ and ESA+ cells confirmed by visual examination under fluorescent microscope. Flow cytometry analysis of CD44+/ESA+ (CSCs) and CD44-/ESA- cells (non-CSCs) sorted from PANCI and PK45 cells with immunofluorescence conjugated antibodies by the FACSAria cell sorter. (B) Colony formation ability of CSCs and non-CSCs delivered from PANCI and PK45 cells. (C) Spheroid formation ability of CSCs and non-CSCs delivered from PANCI and PK45 cells.

StatView software (SAS Institute, Inc., Cary, NC). A p-value less than 0.05 was defined as significant for all comparisons.

\section{Results}

\section{Pancreatic CSC Isolation and Its Properties}

To isolate and confirm the pancreatic cancer stem cell (CSC) properties, we sorted the subpopulation of CD44 +/ESA+ and non-CSC CD44-/ESA- cells from PANC1 and PK45 cells using the FACS Aria cell sorter, and then performed assays for colony and spheroid formation ability. As shown in Figure 1, the CD44+/ESA+ cells have significantly higher numbers to form colonies and more large-sized spheroid compared to that of the CD44-/ESAcells, indicating that the CD44+/ESA+ cells have CSC properties.

\section{Effects of Carbon-Ion Beam Irradiation Alone or in Combination with miR-200c Mimic on Colony Formation Ability of the Pancreatic CSCs}

To investigate the effect of exposure to carbon-ion beam irradiation alone in combination miR-200c mimic on the colony formation ability of pancreatic CSCs and non-CSCs. CSCs and non-CSCs were seeded 


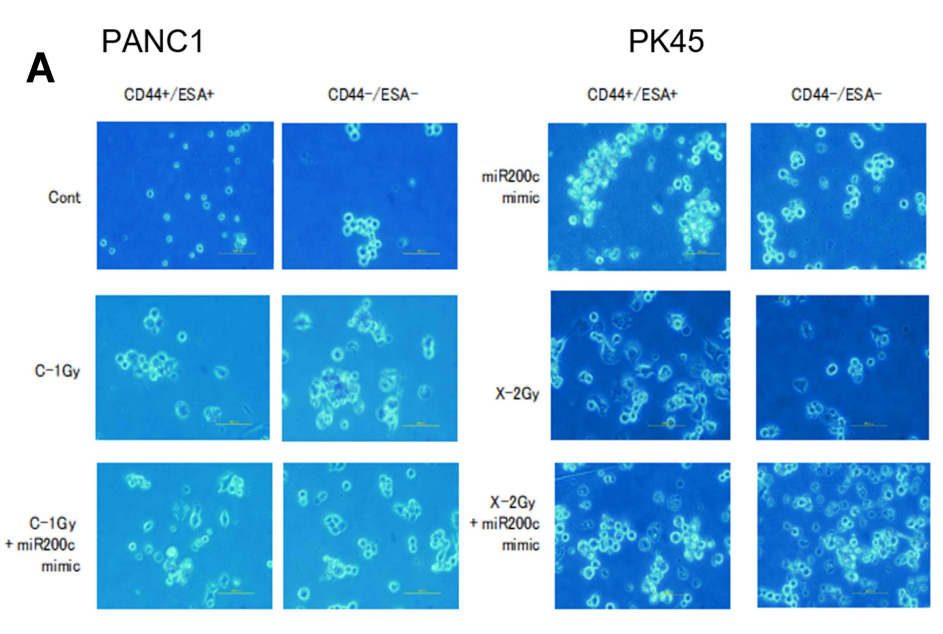

B

PK45
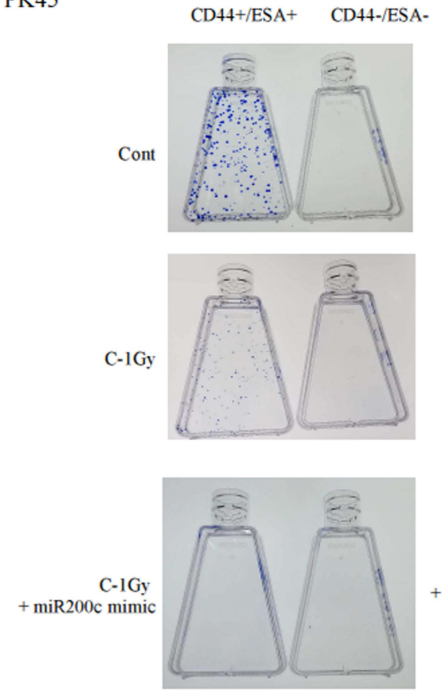

CD44+/ESA+ CD44-/ESA-
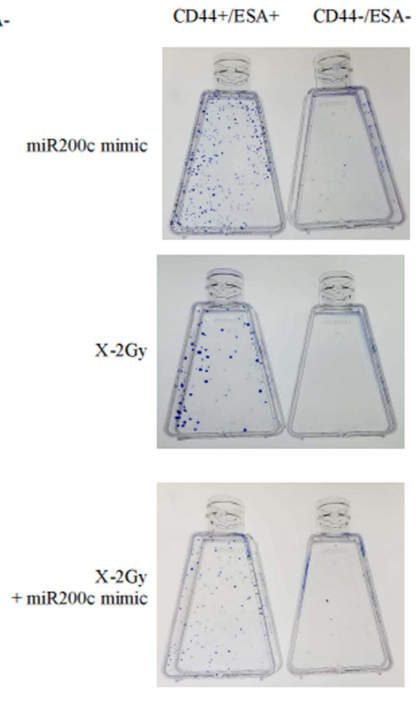

Figure 2 (A) Morphological changes of CSCs and non-CSCs delivered from PK45 cells $72 \mathrm{~h}$ after treatment with carbon-ion beam irradiation alone, X-ray irradiation alone or in combination with the miR-200c mimic. (B) Colony formation of CSCs and non-CSCs delivered from PK45 cells after treatment with carbon-ion beam, X-ray irradiation alone or in combination with the miR-200c mimic. ${ }^{*} p<0.01$ compared to the control.

and incubated for 2 weeks after transfection with miR200c. Figure $2 \mathrm{~A}$ shows morphological changes in the CSCs and non-CSCs $72 \mathrm{~h}$ after exposure to a carbonion beam irradiation (C-1 Gy) alone, X-ray (2 Gy) alone, or the miR-200c mimic (10 $\mathrm{nM})$ alone, or a combination of any of these three treatments. The results showed that the cells were killed by the combination treatment, especially constituting the carbon-ion beam irradiation and miR-200c mimic. As shown in Figure 2B, the colony formation ability of the pancreatic CSCs was decreased after treatment with the carbon-ion beam alone, and the miR-200c mimic alone

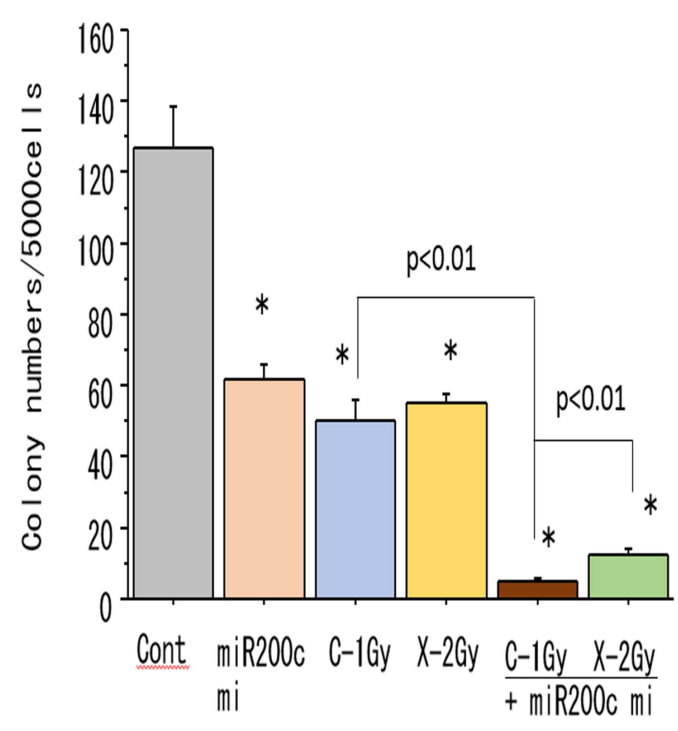

treatment, and it was significantly further decreased by its combination treatment compared to that with $\mathrm{X}$-ray irradiation alone.

\section{Effects of Carbon-Ion Beam Irradiation Alone or in Combination with miR-200c Mimic on Spheroid Formation Ability of the Pancreatic CSCs}

To investigate the spheroid formation ability of the pancreatic CSCs and non-CSCs irradiated with carbon-ion beam alone or in combination with the miR-200c mimic, 
PK45
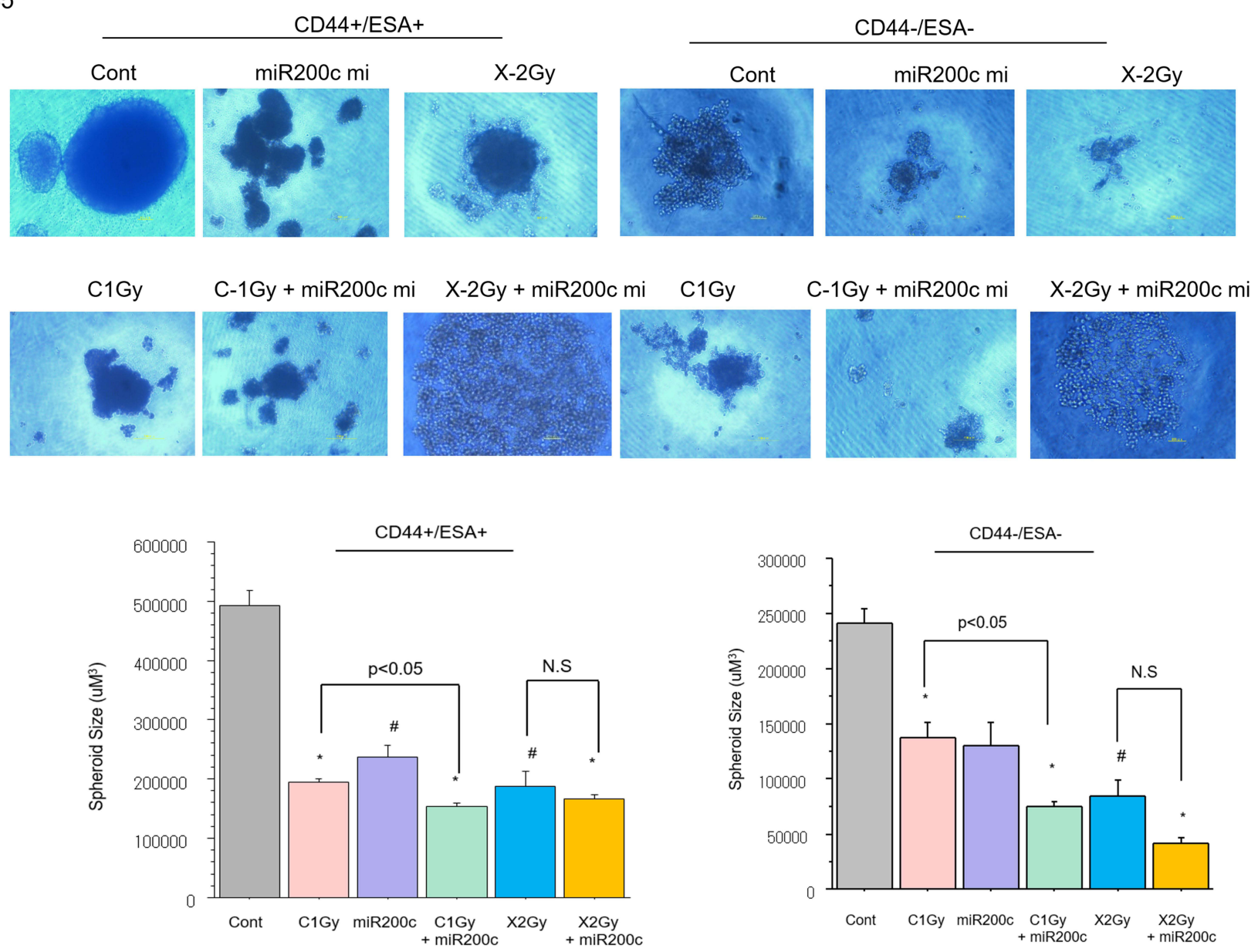

Figure 3 Spheroid formation of CSCs and non-CSCs delivered from PANCI and PK45 cells after treatment with carbon-ion beam irradiation alone or in combination with the miR-200c mimic. \#p<0.05, ${ }_{p}<0.01$ compared to the control.

miR200c transfected CSCs and non-CSCs were placed in 96-well round-bottomed Sumilon Celltight spheroid plates (Sumilon, Sumitomo Bakelite Co., Tokyo) and incubated for 7 days. As shown in Figure 3, the spheroid formation ability of the pancreatic CSCs was slightly inhibited after treatment with either carbon-ion beam alone or miR-200c mimic alone. However, this ability showed a further significant reduction when a combination was applied compared to that with X-ray irradiation alone (Figure 3).

\section{Carbon-lon Beam Irradiation Combined with the miR-200c Mimic Decreases Pancreatic CSC Viability}

A CellTiter-Glo luminescent cell viability assay was performed to examine the effects of the combination of miR200c mimic and the carbon-ion beam irradiation on suppression of pancreatic CSCs and non-CSCs viability. As shown in
Figure 4, the treatment with miR-200c mimic alone significantly inhibited the viability of the pancreatic CSCs, whereas the viability was only slightly inhibited upon treatment with carbon-ion beam irradiation. However, the viability of the pancreatic CSCs was greatly inhibited by the combination treatment of carbon-ion beam irradiation and miR-200c mimic. The same response was observed in non-CSCs.

\section{Effects of Carbon-lon Beam Irradiation Alone or in Combination with miR-200c Mimic on Expression Genes Related to Apoptosis and Autophagy in Pancreatic CSCs}

Quantitative real-time PCR (qPCR) was performed to investigate whether carbon-ion beam irradiation alone or in combination with miR-200c mimic and/or GEM on the effects of the expression of apoptosis-related genes. As 


\section{PK45 Viability $72 \mathrm{~h}$}

CD44-/ESA-

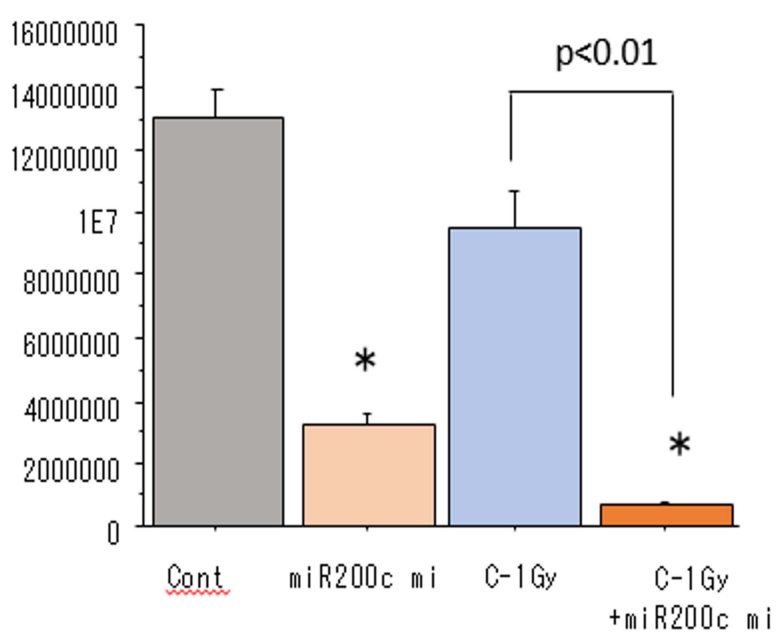

CD44+/ESA+

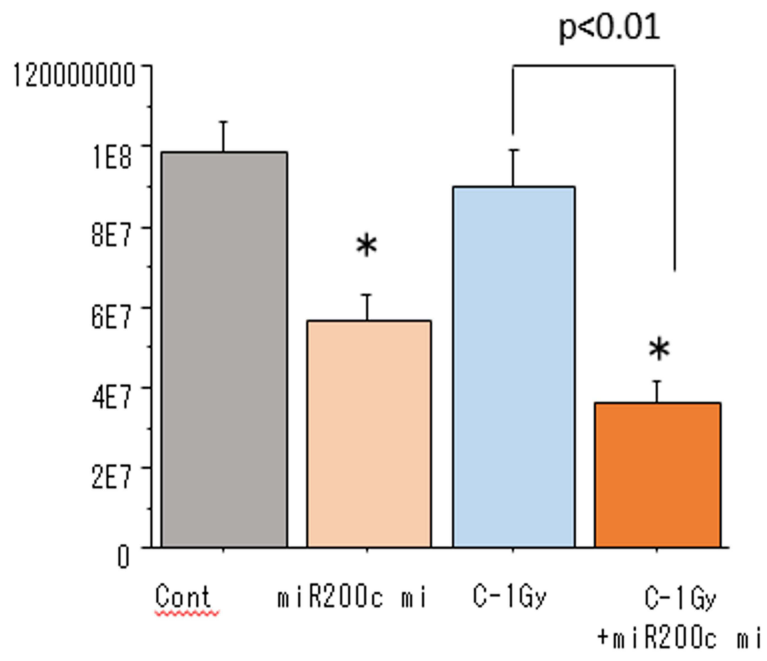

Figure 4 Cell viability of non-CSCs and CSCs delivered from PK45 cells after treatment with carbon-ion beam irradiation alone or in combination with the miR-200c mimic. ${ }^{*} \mathrm{p}<0.01$ compared to the control.

shown in Figure 5A, expression of the apoptosis-related gene Bax was increased after exposure to carbon-ion beam irradiation alone but not in the presence of the miR-200c mimic. This Bax expression was significantly increased after treatment with carbon-ion beam irradiation combined with miR-200c mimic, and enormously enhanced by the additional treatment of plus GEM in the pancreatic CSCs. The expression of the anti-apoptotic gene $\mathrm{Bcl} 2$ was also increased by carbon-ion beam irradiation alone, and their combination treatment. However, no significant changes were observed by the additional treatment of GEM in the pancreatic CSCs. In conclusion, Bax gene expression was increased only by carbon-ion beam irradiation in combination with miR-200c mimic, and the $B c l 2$ gene expression was increased by the triple combination treatment of carbon-ion beam irradiation, miR-200c mimic and GEM in the pancreatic non-CSCs.

The expression of autophagy-related genes, Beclin- 1 and p62, was increased after carbon-ion beam irradiation alone. A further significant increase was caused by triple combination treatment of carbon-ion beam irradiation, miR-200c mimic and GEM in the pancreatic CSCs. In contrast, Beclin-1 expression was increased by treatment with carbonion beam irradiation alone, or in combination with miR-200c mimic or triple combination of plus GEM. However, the p62 expression was increased by all treatment combinations in the pancreatic non-CSCs (Figure 5B).

\section{Effects of Carbon-lon Beam Irradiation Alone or in Combination with miR-200c Mimic on Expression of Angiogenesis-Related Genes in Pancreatic Cancer Stem Cells}

To investigate the expression of exposure to carbon-ion beam irradiation alone or in combination with miR-200c mimic and/or GEM on expression of angiogenesis-related genes, the expression of the HIF 1a and VEGF was determined by qPCR. As seen from Figure 5C, the HIF1a expression was significantly inhibited by carbon-ion beam irradiation combined with miR-200c mimic compared to miR-200c mimic treatment alone or miR-200c mimic combined with GEM treatment in pancreatic CSCs. The VEGF expression increased after exposure to carbonion beam irradiation alone, miR-200c mimic and GEM alone or its combination treatment in pancreatic CSCs.

\section{Effects of Carbon-lon Beam Irradiation Alone or in Combination with miR-200c Mimic on Morphological and Histopathological Changes in Pancreatic Xenograft Tumors}

To examine the effect of carbon-ion beam irradiation treatment alone or in combination with miR-200c 
A
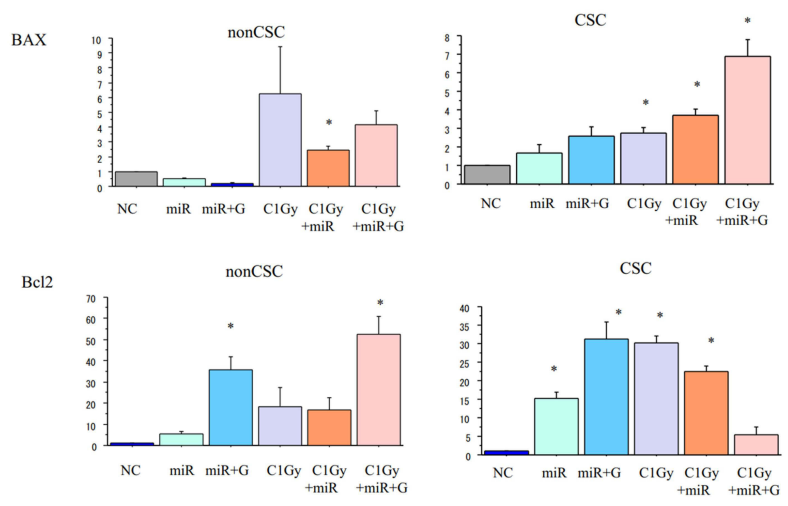

C
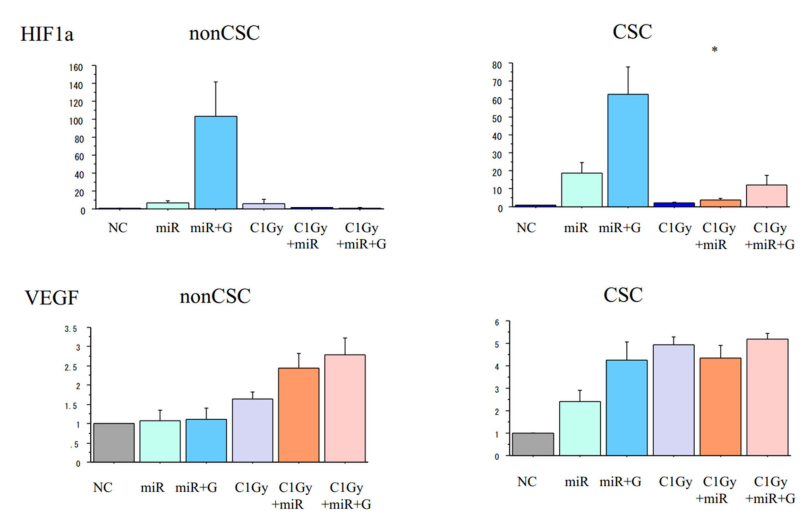

B
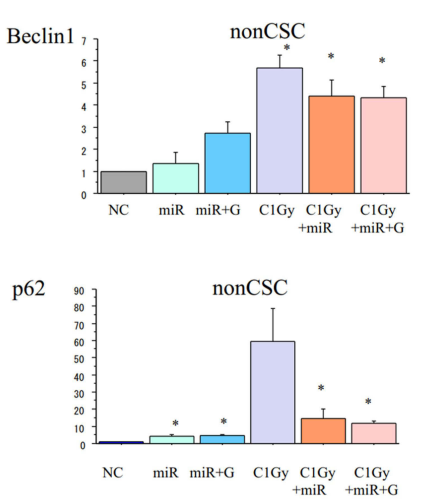
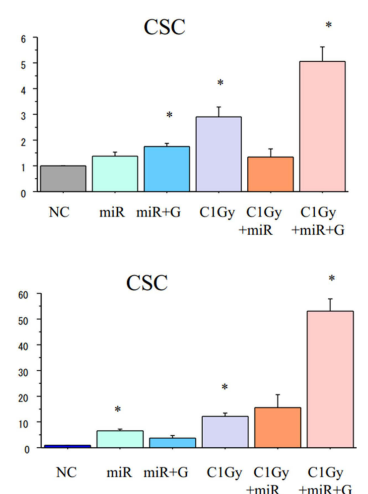

Figure 5 (A) Changes in the expression of apoptosis-related genes $96 \mathrm{~h}$ after treatment with carbon-ion beam irradiation alone or in combination with the miR-200c mimic and/or gemcitabine in CSCs and non-CSCs delivered from PK45 cells. (B) Changes in the expression of autophagy-related genes $96 \mathrm{~h}$ after treatment with carbon-ion irradiation alone or in combination with the miR-200c mimic and/or gemcitabine in CSCs and non-CSCs delivered from PK45 cells. (C) Expression changes of angiogenesisrelated genes $96 \mathrm{~h}$ after treatment with carbon-ion irradiation alone or in combination with the miR-200c mimic and/or gemcitabine in CSCs and non-CSCs delivered from PK45 cells. ${ }^{*} \mathrm{p}<0.01$ compared to the control.

mimic on the xenograft tumor growth and associated histopathological changes, we injected PK45 cells into NOD-SCID mice and then treated the tumors after they had grown to a certain size $(7-8 \mathrm{~mm}$ diameter). As shown in Figure $6 \mathrm{~A}$ and $\mathrm{B}$, treatment with $15 \mathrm{~Gy}$ of carbon-ion beam irradiation in combination with the miR-200c mimic predominantly suppressed the tumor growth compared to that obtained by treatment with miR-200c alone and 30 Gy of carbon-ion beam irradiation alone 1 month after treatment. Two months after treatment, the tumor growth was still regressed by 15 Gy of carbon-ion beam irradiation in combination with the miR-200c mimic. Interestingly, the tumor growth was further regressed by 30 Gy of carbon ion beam irradiation alone, but the tumors were regrown in the miR200c mimic-alone treatment group. Histopathological analysis showed that combined treatment with carbon-ion beam and the miR-200c mimic effectively destroyed the pancreatic xenograft tumor cells with significant tumor cell necrosis, cavitation and fibrosis compared to the miR-200c mimic-alone treatment. High-dose carbon ion beam irradiation appears to induce more severe tumor cell cavitation (Figure 6C).

\section{Discussion}

We found that the viability as well as colony and spheroid formation abilities of the pancreatic CSCs decreased after treatment with carbon-ion beam alone, and miR-200c mimic alone. These abilities were significantly decreased by the combination treatment, whereas X-ray irradiation alone could cause only slight inhibition. This finding indicated that the miR-200c mimic effectively enhanced the carbon-ion radiosensitivity in the pancreatic CSCs. This is partially in line with previous reports that miR-200c increased the radiosensitivity in human cancer cells by 

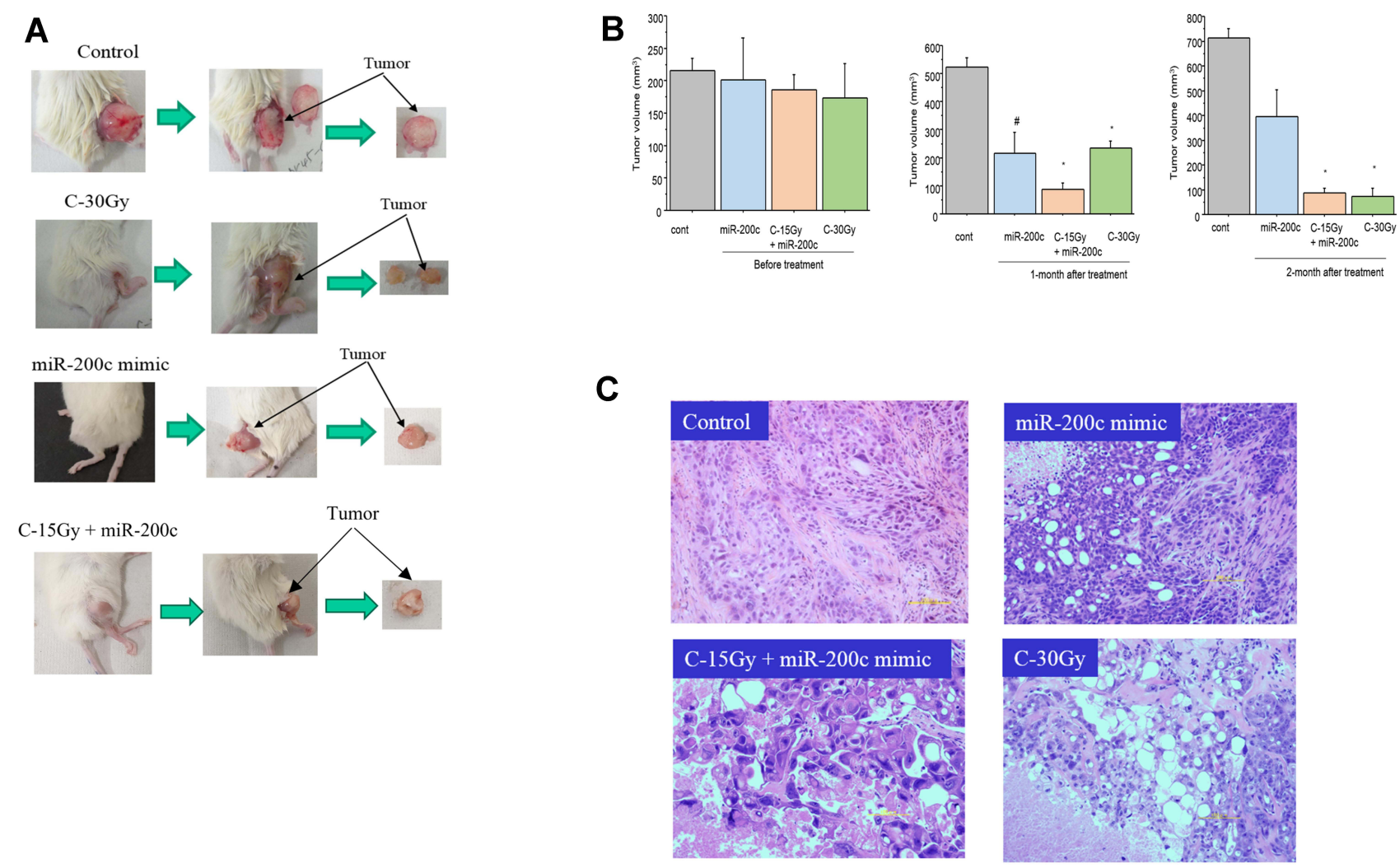

Magnification x 200

Figure 6 (A) Morphological changes of PK45 xenograft tumor after 30 Gy of carbon ion beam irradiation alone, miR-200c mimic alone or 15 Gy of carbon ion beam irradiation in combination with the miR-200c mimic. (B) Regression of PK45 xenograft tumor before and after treatment with $30 \mathrm{~Gy}$ of carbon ion beam irradiation alone, miR-200c mimic alone or 15 Gy of carbon ion beam irradiation in combination with the miR-200c mimic. \#p<0.05, * $<<0.01$ compared to the Control. (C) Histopathological changes in the PK45 xenograft tumor after $30 \mathrm{~Gy}$ of carbon ion beam irradiation alone, miR-200c mimic alone or 15 Gy of carbon ion beam irradiation in combination with the miR-200c mimic.

downregulating p-EGFR and p-AKT and inducing apoptosis and autophagy. ${ }^{41-44}$

It has been reported that miR-200c expression is downregulated in breast CSCs, and the miR-200c mimic effectively increases breast cancer cell chemosensitivity and inhibits stemness and xenograft tumor growth. ${ }^{27-35}$ Pancreatic cancer patients with a higher expression of miR-200c showed a close correlation with low invasion ability and better prognosis. ${ }^{48}$ The miR-200c mimic decreased pancreatic CSC colony formation, invasion and chemoresistance. ${ }^{29,49}$ In this study, apoptosis-related Bax expression was increased after exposure to carbon-ion beam irradiation alone but not by the miR-200c mimic alone. However, Bax expression was significantly increased after the carbon-ion beam irradiation was combined with miR-200c mimic, and enormously enhanced by triple combination treatment with GEM in the pancreatic CSCs. In comparison, the expression of the anti-apoptotic gene $\mathrm{Bcl} 2$ was also increased by the exposure to carbonion beam irradiation alone, miR-200c mimic alone or its combination treatment. However, it was not significantly changed by the triple combination treatment of these agents in the pancreatic CSCs. These data suggest that the Bax gene expression was increased only by carbonion beam irradiation in combination with miR-200c mimic. However, Bcl2 gene expression was increased by the triple combination treatment of carbon-ion beam irradiation, miR-200c mimic and GEM in pancreatic nonCSCs. This is partially consistent with previous reports that miR-200c mimic increased the caspase-3 and Bax expression and decreased the $\mathrm{Bcl} 2$ expression in human glioblastoma and gastric cancer cells. ${ }^{39,50,51}$

In this study, expression of autophagy-related genes, Beclin-1 and p62, was increased after carbon-ion beam irradiation alone, a further significant increase was obtained by the additional treatment of GEM in the pancreatic CSCs. A previous report showed that miR-200c inhibited radiation-induced autophagy-related proteins such as LC3AB and p62 in breast cancer cells. ${ }^{44}$ In contrast, Beclin-1 expression was increased by exposure to 
carbon-ion beam irradiation alone, or in combination with miR-200c mimic or the triple combination with GEM. However, p62 expression was increased by all treatment combinations in pancreatic non-CSCs. miR-200c has the potential to suppress HIF-1 $\alpha / \mathrm{VEGF}$ expression in bladder cancer cells and inhibit angiogenesis, and these regulations were achieved by targeting Akt2/mTOR. ${ }^{22}$ The investigation on the expression of angiogenesis-related genes, HIF1a and VEGF showed that HIF1a expression was significantly inhibited by carbon-ion beam irradiation combined with miR-200c mimic, but not by its isolated treatment in the pancreatic CSCs. VEGF expression was increased by the treatment with carbon-ion beam irradiation alone, miR-200c mimic and GEM alone or its combination treatment in pancreatic CSCs. This finding is partially consistent with a previous report that the miR200c mimic inhibits the expression of HIF1a, MMP2, VEGF and E-cadherin in human glioblastoma cells. ${ }^{44,45}$

Currently, several therapeutic miRNA delivery systems, ${ }^{58,59}$ such as virus-based delivery, ${ }^{60}$ non-viral delivery (artificial lipid-based vesicles, ${ }^{4,61-64}$ polymerbased $^{65}$ or chemical structures, ${ }^{66}$ and extracellular vesiclebased delivery systems ${ }^{67,68}$ have been reported. Reid et al reported that delivery of miR-16 using EDV'M nanocell platform effectively suppressed xenograft malignant pleural mesothelioma in vivo. ${ }^{61}$ Coetez et al reported that therapeutic delivery of miR-200c mimics using liposomal nanoparticle effectively enhanced radiosensitivity in xenograft lung tumor in vivo, ${ }^{43}$ implying that those of delivery systems may be useful in clinical trial in the future.

In the present study, relatively a low dose of carbon-ion beam irradiation (15 Gy) combined with miR-200c mimic or with high dose of carbon-ion beam irradiation (30 Gy) alone greatly suppressed tumor growth compared to that with miR-200c-alone treatment. This finding suggests that combination with miR-200c mimic can enhance the radiosensitivity of pancreatic tumor cell to carbon ion beams in accompanied with significant tumor cell necrosis, cavitation and fibrosis.

In summary, the combination treatment with carbonion beam irradiation and miRNA-200c mimic has advantage to eliminate pancreatic cancer cells containing CSCs accompanied with elevated expression of apoptotic- and autophagic-related genes, Bax, Beclin-1, and p62 and decreased expression of angiogenesis-related HIF $1 \alpha$ in vitro and destructed tumor cells in vivo compared to carbon-ion beam irradiation alone.

\section{Abbreviations}

CIRT, carbon-ion radiotherapy; miRNAs, microRNAs; CSC, cancer stem cell; DMEM, Dulbecco's modified Eagle's medium; FBS, fetal bovine serum; HIMAC, heavy ion medical accelerator in Chiba; NIRS; National Institute of Radiological Sciences; QST, National Institutes for Quantum and Radiological Science and Technology; GEM, gemcitabine; LET, linear energy transfer.

\section{Acknowledgments}

This work was partially supported by Japan-Korea Bilateral Programs Joint Research Projects to S Sai (No.17032211-000542) and EH Kim (NRF2019M2A 2B4095150), and Pancreas Research Foundation of Japan (to $\mathrm{G}$ Vares). We are grateful to Mr Yamada $\mathrm{T}$ for his kind technical support.

\section{Disclosure}

The authors report no conflicts of interest in this work.

\section{References}

1. Reames BN, Blair AB, Krell RW, et al. Management of locally advanced pancreatic cancer: results of an international survey of current practice. Ann Surg. 2019;273(6):1173-1181.

2. Ruarus A, Vroomen L, Puijk R, Scheffer H, Meijerink M. Locally advanced pancreatic cancer: a review of local ablative therapies. Cancers (Basel). 2018;10(1):16.

3. Gemenetzis G, Groot VP, Blair AB, et al. Survival in locally advanced pancreatic cancer after neoadjuvant therapy and surgical resection. Ann Surg. 2019;270(2):340-347.

4. Lambert A, Schwarz L, Borbath I, et al. An update on treatment options for pancreatic adenocarcinoma. Ther Adv Med Oncol. 2019; 11:1758835919875568.

5. Goto Y, Nakamura A, Ashida R, et al. Clinical evaluation of intensity-modulated radiotherapy for locally advanced pancreatic cancer. Radiat Oncol. 2018;13:118.

6. Stefanowicz S, Stützer K, Zschaeck S, Annika Jakobi EG, Troost C. Comparison of different treatment planning approaches for intensity-modulated proton therapy with simultaneous integrated boost for pancreatic cancer. Radiat Oncol. 2018;13(1):228.

7. Lambert A, Schwarz L, Borbath I, et al. An update on treatment options for pancreatic adenocarcinoma. Ther Adv Med Oncol. 2019;25(11):1758835919875568.

8. Loehrer PJ, Sr YF, Cardenes H, et al. Gemcitabine alone versus gemcitabine plus radiotherapy in patients with locally advanced pancreatic cancer: an eastern cooperative oncology group trial. J Clin Oncol. 2011;29(31):4105-4112.

9. Shalini Moningi AS, Dholakia SP, Raman AB, et al. The role of stereotactic body radiation therapy for pancreatic cancer: a single-institution experience. Ann Surg Oncol. 2015;22(7):23 52-2358.

10. Tozzi A, Comito T, Alongi F, et al. SBRT in unresectable advanced pancreatic cancer: preliminary results of a mono-institutional experience. Radiat Oncol. 2013;8:148.

11. Herman JM, Chang DT, Goodman KA, et al. Phase 2 multi-institutional trial evaluating gemcitabine and stereotactic body radiotherapy for patients with locally advanced unresectable pancreatic adenocarcinoma. Cancer. 2015;121(7):1128-1137. 
12. Krishnan S, Chadha AS, Suh Y, et al. Focal radiation therapy dose escalation improves overall survival in locally advanced pancreatic cancer patients receiving induction chemotherapy and consolidative chemoradiation. Int J Radiat Oncol Biol Phys. 2016;94 (4):755-765.

13. Ben-Josef E, Schipper M, Francis IR, et al. A Phase I/II trial of intensity-modulated radiation (IMRT) dose escalation with concurrent fixed- dose rate gemcitabine (FDR-G) in patients with unresectable pancreatic cancer. Int J Radiat Oncol Biol Phys. 2012;84 (5):1166-1171.

14. Nichols RC, Huh S, Zuofeng L, Rutenberg M. Proton therapy for pancreatic cancer. World J Gastrointest Oncol. 2015;7(9):141-147.

15. Liermann J, Shinoto M, Syed M, Debus J, Herfarth K, Naumann P. Carbon ion radiotherapy in pancreatic cancer: a review of clinical data. Radiother Oncol. 2020;19(147):145-215.

16. Shinoto M, Yamada S, Terashima K, et al.; Working Group for Pancreas Cancer. Carbon ion radiation therapy with concurrent gemcitabine for patients with locally advanced pancreatic cancer Int J Radiat Oncol Biol Phys. 2016;95(1):498-504.

17. Kawashiro S, Yamada S, Isozaki Y, Nemoto K, Tsuji H, Kamada T. Carbon-ion radiotherapy for locoregional recurrence after primary surgery for pancreatic cancer. Radiother Oncol. 2018;129 (1):101-104.

18. Kawashiro S, Yamada S, Okamoto M, et al. Multi-institutional study of carbon-ion radiotherapy for locally advanced pancreatic cancer: Japan Carbon-ion Radiation Oncology Study Group (J-CROS) study 1403 pancreas. Int $J$ Radiat Oncol Biol Phys. 2018;101 (5):1212-1221.

19. Sai S, Wakai T, Vares G, et al. Combination of carbon ion beam and gemcitabine causes unreparable DNA damage and death of radioresistant pancreatic cancer stem-like cells in vitro and in vivo. Oncotarget. 2015;6:5517-5535.

20. Kong YW, Ferland-McCollough D, Jackson TJ, et al. microRNAs in cancer management. Lancet Oncol. 2012;13(6):e249-58.

21. Czochor JR, Glazer PM. microRNAs in cancer cell response to ionizing radiation. Antioxid Redox Signal. 2014;21(2):293-312.

22. Rodriguez A, Griffiths-Jones S, Ashurst JL, et al. Identification of mammalian microRNA host genes and transcription units. Genome Res. 2004;14(10A):1902-1910.

23. Cullen BR. Transcription and processing of human microRNA precursors. Mol Cell. 2004;16(6):861-865.

24. Takahashi RU, Prieto-Vila M, Kohama I, et al. Development of miRNA-based therapeutic approaches for cancer patients. Cancer Sci. 2019;110(4):1140-1147.

25. Vares G, Ahire V, Sunada S, et al. A multimodal treatment of carbon ions irradiation, miRNA-34 and mTOR inhibitor specifically control high-grade chondrosarcoma cancer stem cells. Radiother Oncol. 2020;S0167-8140:20.

26. Kim EH, Kim JY, Kim MS, et al. Molecular mechanisms underlying the enhancement of carbon ion beam radiosensitivity of osteosarcoma cells by miR-29b. Am J Cancer Res. 2020;10(12):4357-4371.

27. Ma C, Huang T, Ding YC, et al. MicroRNA-200c overexpression inhibits chemoresistance, invasion and colony formation of human pancreatic cancer stem cells. Int J Clin Exp Pathol. 2015;8 (6):6533-6539.

28. Byun Y, Choi YC, Jeong Y, et al. MiR-200c downregulates HIF-1a and inhibits migration of lung cancer cells. Cell Mol Biol Lett. 2019;24:28.

29. Yu J, Ohuchida K, Mizumoto K, et al. MicroRNA, hsa-miR-200c, is an independent prognostic factor in pancreatic cancer and its upregulation inhibits pancreatic cancer invasion but increases cell proliferation. Mol Cancer. 2010;9:169. doi:10.1186/1476-45989-16

30. Lo WL, Yu CC, Chiou GY, et al. MicroRNA-200c attenuates tumour growth and metastasis of presumptive head and neck squamous cell carcinoma stem cells. $J$ Pathol. 2011;223(4):482-495.
31. Tang H, Song C, Ye F, et al. miR-200c suppresses stemness and increases cellular sensitivity to trastuzumab in HER2+ breast cancer. J. Cell Mol Med. 2019;23(12):8114-8127.

32. Rahimi M, Sharifi-Zarchi A, Zarghami N, Geranpayeh L, Ebrahimi M, Alizadeh E. Down-regulation of miR-200c and up-regulation of miR-30c target both stemness and metastasis genes in breast cancer. Cell J. 2020;21(4):467-478.

33. Karimi Dermani F, Amini R, Saidijam M, Najafi R. miR-200c, a tumor suppressor that modulate the expression of cancer stem cells markers and epithelial-mesenchymal transition in colorectal cancer. J Cell Biochem. 2018;119(7):6288-6295.

34. Park SM, Gaur AB, Lengyel E, Peter ME. The miR-200 family determines the epithelial phenotype of cancer cells by targeting the E-cadherin repressors ZEB1 and ZEB2. Genes Dev. 2008;22 (7):894-907.

35. Shimono Y, Zabala M, Cho RW, et al. Downregulation of miRNA-200c links breast cancer stem cells with normal stem cells. Cell. 2009;138(3):592-603.

36. Hambardzumyan D, Squartro M, Holland EC. Radiation resistance and stem-like cells in brain tumors. Cancer Cell. 2006;10:454-456.

37. Rich JN. Cancer stem cells in radiation resistance. Cancer Res. 2007;67:8980-8984).

38. Dingli D, Michor F. Successful therapy must eradicate cancer stem cells. Stem Cells. 2006;24:2603-2610.

39. Vermeulen L, de Sousa e Melo F, Richel DJ, Medema JP. The developing cancer stem-cell model: clinical challenges and opportunities. Lancet Oncol. 2012;13:e83-e89.

40. Wang B, Zheng J, Li R, et al. Long noncoding RNA LINC02582 acts downstream of miR-200c to promote radioresistance through CHK1 in breast cancer cells. Cell Death Dis. 2019;Oct(10):764.

41. Koo T, Cho BJ, Kim DH, et al. MicroRNA-200c increases radiosensitivity of human cancer cells with activated EGFR-associated signaling. Oncotarget. 2017;8(39):65457-65468.

42. Sun Q, Liu T, Yuan Y, et al. MiR-200c inhibits autophagy and enhances radiosensitivity in breast cancer cells by targeting UBQLN1. Int J Cancer. 2015;136(5):1003-1012.

43. Cortez MA, Valdecanas D, Zhang X, et al. Therapeutic delivery of miR-200c enhances radiosensitivity in lung cancer. Mol Ther. 2014;22(8):1494-1503.

44. Lin J, Liu C, Gao F, et al. miR-200c enhances radiosensitivity of human breast cancer cells. J Cell Biochem. 2013;114(3):606-615.

45. Shi L, Zhang S, Wu H, et al. MiR-200c increases the radiosensitivity of non-small-cell lung cancer cell line A549 by targeting VEGF-VEGFR2 pathway. PLoS One. 2013;8(10):e78344.

46. Feng ZM, Qiu J, Chen XW, et al. Essential role of miR-200c in regulating self-renewal of breast cancer stem cells and their counterparts of mammary epithelium. BMC Cancer. 2015;15:645.

47. Lim YY, Wright JA, Attema JL, et al. Epigenetic modulation of the miR-200 family is associated with transition to a breast cancer stem-cell-like state. $J$ Cell Sci. 2013;126(Pt 10):2256-2266.

48. Jiang T, Dong P, Li L, et al. MicroRNA-200c regulates cisplatin resistance by targeting ZEB2 in human gastric cancer cells. Oncol Rep. 2017;38(1):151-158.

49. Wei W, Shi L, Chen W, et al. miR-200c regulates the proliferation, apoptosis and invasion of gastric carcinoma cells through the downregulation of EDNRA expression. Int $J$ Mol Med. 2018;41 (3):1619-1626.

50. Guo E, Wang Z, Wang S. MiR-200c and miR-141 inhibit ZEB1 synergistically and suppress glioma cell growth and migration. Eur Rev Med Pharmacol Sci. 2016;20(16):3385-3391.

51. Yuan D, Zheng S, Wang L, et al. MiR-200c inhibits bladder cancer progression by targeting lactate dehydrogenase A. Oncotarget. 2017;8(40):67663-67669.

52. Cui X, Oonishi K, Tsujii H, et al. Effects of carbon ion beam on putative colon cancer stem cells and its comparison with X-ray. Cancer Res. 2011;71:3676-3687. 
53. Oonishi K, Cui X, Hirakawa H, et al. Different effects of carbon ion beams and X-rays on clonogenic survival and DNA repair in human pancreatic cancer stem-like cells. Radiother Oncol. 2012;105:258-265.

54. Sai S, Vares G, Kim EH, et al. Carbon ion beam combined with cisplatin effectively disrupts triple negative breast cancer stem-like cells in vitro. Mol Cancer. 2015;14:166. doi:10.1186/s12943-0150429-7

55. Sai S, Suzuki M, Kim EH, Hayahsi M, Yamamoto N, Miyamoto T. Effects of carbon ion beam alone or in combination with cisplatin on malignant mesothelioma cells in vitro. Oncotarget. 2018;9(19): 14849-14861.

56. Kim EH, Kim MS, Takahashi A, et al. Carbon-ion beam irradiation alone or in combination with zoledronic acid effectively kills osteosarcoma cells. Cancers. 2020;12:698.

57. Sai S, Kim EH, Suzuki M, Horimoto Y, Hayashi M. Combination of carbon-ion beam and dual tyrosine kinase inhibitor, lapatinib, effectively destroys HER2 positive breast cancer stem-like cells. Am $J$ Cancer Res. 2020;10(8):2371-2386.

58. Reid G, Kao SC, Pavlakis N, et al. Clinical development of TargomiRs, a miRNA mimic-based treatment for patients with recurrent thoracic cancer. Epigenomics. 2016;Aug(8):1079-1085.

59. Forterre A, Komuro H, Aminova S, Harada M. A comprehensive review of cancer MicroRNA therapeutic delivery strategies. Cancers (Basel). 2020;12(7):1852. doi:10.3390/cancers 12071852
60. Bai Z, Wei J, Yu C, et al. Non-viral nanocarriers for intracellular delivery of microRNA therapeutics. J Mater Chem B. 2019;7: 1209-1225.

61. Mansoori B, Silvestris N, Mohammadi A, et al. miR-34a and miR-200c have an additive tumor-suppressive effect on breast cancer cells and patient prognosis. Genes (Basel). 2021;12(2):267.

62. Reid G, Pel ME, Kirschner MB, et al. Restoring expression of miR-16: a novel approach to therapy for malignant pleural mesothelioma. Ann Oncol. 2013;24(12):3128-3135.

63. Bushman FD. Retroviral integration and human gene therapy. J Clin Invest. 2007;117:2083-2086.

64. Kaban K, Salva E, Akbuga J. The effects of chitosan/miR-200c nanoplexes on different stages of cancers in breast cancer cell lines. Eur J Pharm Sci. 2016;95:103-110.

65. Fernandez CA, Rice KG. Engineered nanoscaled polyplex gene delivery systems. Mol Pharm. 2009;6:1277-1289.

66. Ochiya T, Nagahara S, Sano A, Itoh H, Terada M. Biomaterials for gene delivery: atelocollagen-mediated controlled release of molecular medicines. Curr Gene Ther. 2001;1:31-52.

67. Tkach M, Théry C. Communication by extracellular vesicles: where we are and where we need to go. Cell. 2016;164:1226-1232.

68. EL Andaloussi S, Mäger I, Breakefield XO, Wood MJ. Extracellular vesicles: biology and emerging therapeutic opportunities. Nat Rev Drug Discov. 2013;12:347-357.

\section{Publish your work in this journal}

OncoTargets and Therapy is an international, peer-reviewed, open access journal focusing on the pathological basis of all cancers, potential targets for therapy and treatment protocols employed to improve the management of cancer patients. The journal also focuses on the impact of management programs and new therapeutic agents and protocols on patient perspectives such as quality of life, adherence and satisfaction. The manuscript management system is completely online and includes a very quick and fair peer-review system, which is all easy to use. Visit http://www.dovepress.com/ testimonials.php to read real quotes from published authors. 